\title{
LES GROUPEMENTS DE MACROPHYTES AQUATIQUES DE LA LOUE
}

par R. BONNARD ${ }^{1}$ et A. MICHON ${ }^{2}$

\begin{abstract}
Les groupements de macrophytes aquatiques de la Loue ont été déterminés à partir de relevés effectués en avril, mai et juin 1977. Parallèlement, les variations de trois composantes morphodynamiques, à savoir la vitesse du courant, la nature du substrat et la hauteur d'eau, semblant régir la distribution des végétaux, ont été notés.

Cinq associations à phanérogames, Phragmitetum communis, Helosciadetum nodiflori, Callitricho-Myriophylletum alterniflori, Potamogetonetum perfoliatilucentis et Ranunculetum fluitantis ainsi que trois associations à Bryophytes, Cratoneuretum commutati, Cinclidotetum fontinalis et Cinclidotetum aquatici, ont été repérées et leur amplitude typologique déterminée.
\end{abstract}

\section{The groupings of aquatic macrophytes in the River Loue.}

The groupings of aquatic macrophytes of the Loue have been determined from samples taken in April, May and June 1977. Records were also taken simultaneously of three morphodynamic factors that appeared to affect the distribution of the vegetation; namely current speed, the nature of the substratum, and the water depth.

Five associations of flowering plants (Phragmitetum communis, Helosciadetum nodiflori, Callitriche-Myriophylletum alterniflori, Potamogetum perfoliatilucentis and Ranunculetum fluitantis), as well as three associations of bryophtes (Cratoneuretum commutati, Cinclidotetum fontinalis and Cinclidotetum aquatici) have been located and their typological range determined.

\section{INTRODUCTION}

Si la distribution des végétaux aquatiques par rapport à la hauteur d'eau (Hélophytes, Hydrophytes) est relativement bien connue (Sell, 1965), la répartition des groupements végétaux des eaux courantes, pour lesquels la hauteur d'eau n'est pas la seule composante, est loin d'être rigoureusement établie ; en outre doivent être prises en considération la granulométrie du substrat et la vitesse du courant, para-

1. Laboratoire d'Hydroécologie - Division Qualité des Eaux, Pêche et Pisciculture du C.E.M.A.G.R.E.F., 3, quai Chauveau, 69009 Lyon.

2. Bureau d'études : Environnement - Participation - Aménagement, 13, rue des Cordeliers, 39000 Lons-le-Saunier.

Nous remercions M. J. Barbe d'avoir bien voulu critiquer ce manuscrit. 
mètres jouant un rôle important dans la fixation des Macrophytes. Le déterminisme écologique des Bryophytes et des algues benthiques a été abordé par Empain (1973-1974) et Descy (1973-1976) uniquement par leur fréquence d'émersion et leurs relations avec la pollution.

Nous proposons, par le présent travail, d'examiner les groupements végétaux de Phanérogames et de Bryophytes en les reliant aux trois composantes principales de leur déterminisme.

\section{I. — LE MILIEU}

Le cadre de cette étude est constitué par une rivière jurassienne à Salmonidés : La Loue. Née à 595 mètres d'altitude, sur la commune d'Ouhans, la Loue draine une partie du Jura Franc-Contois et joint le Doubs en aval de Dole après 126 kilomètres de cours.

Les travaux de Fournier (1900), Dreyfuss, Théobald et Thiebaut (1962) fournissent une vue d'ensemble de la géologie et de l'hydrologie du bassin versant de la Loue.

Pour la climatologie, nous nous référons aux publications de Weimer-Chofardet (1946), Baillaud (1955), Tronchet (1955) et Delarozière (1968) qui résument un climat soumis à deux influences :

- l'une océanique, marquée par de fortes pluviosités (1200 à

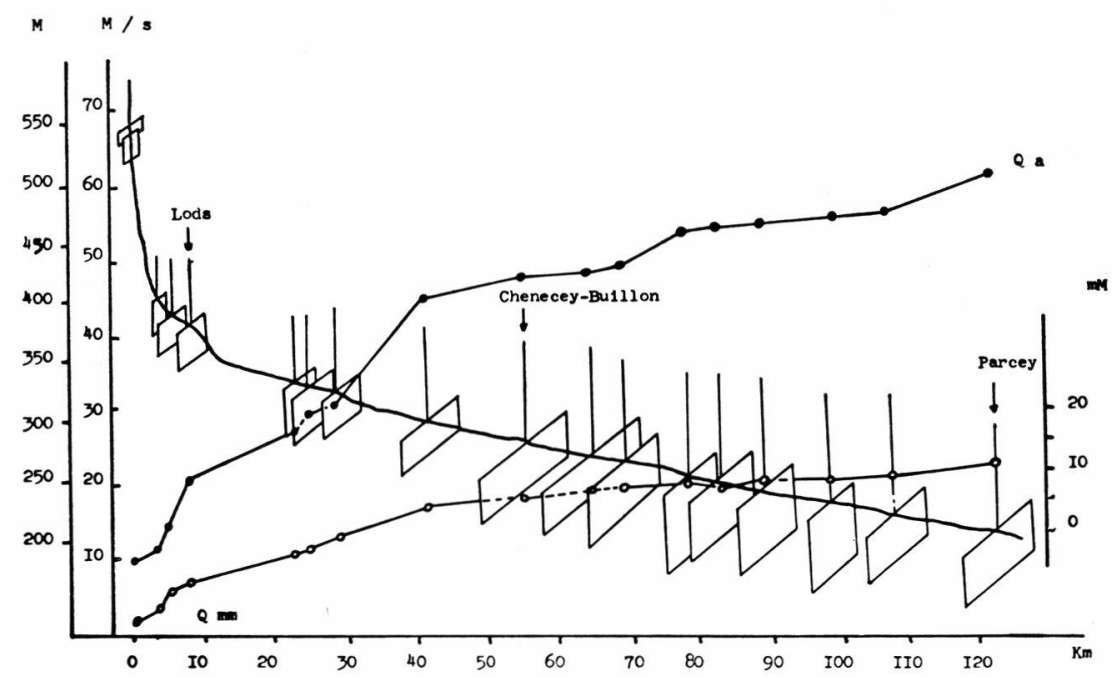

Fig. 1. - La Loue, profils topographiques, hydrologiques et thermiques (d'après Verneaux et Rezzouk 1974).

Qa : débit moyen annuel.

Qmm : débit minimum mensuel.

$\theta \mathrm{mM}$ : température moyenne maximum mensuelle. 
$1500 \mathrm{~mm}$ par an) réparties principalement en automne et au printemps,

- l'autre, continentale, caractérisée par des hivers rigoureux, des étés relativement chauds, et de fortes amplitudes annuelles.

A l'instar de Verneaux (1973) et Verneaux et Rezzouk (1974), nous distinguerons la Haute Loue (de la source à Lods), la Moyenne Loue (de Lods à Chenecey-Buillon) et la Basse Loue de Chenecey-Buillon à Parcey (fig. 1).

\section{2. — LES METHODES}

\subsection{Analyse de la végétation}

Les relevés floristiques ont été établis suivant la technique de l'école Zuricho-Montpellieraine, mise au point par Braun-Blanquet (1964). Les principes sont rigoureusement décrits dans l'ouvrage de Guinochet (1973). Nous rappelons que :

- le choix de l'emplacement des relevés n'est pas fait au hasard, ou choisi arbitrairement, mais c'est un échantillon subjectif lié à l'intuition et aux variations des conditions écologiques;

- la notion de surface floristiquement homogène : "C'est une surface n'offrant pas d'écarts de composition floristique appréciables entre ses différentes parties"(Guinochet 1973). Elle peut être facilement décelée au moyen de la courbe aire-espèce.

- la notion d'individu d'association : "C'est une surface de végétation représentative sur le terrain d'une association végétale » (Allorge 1922). Dans le milieu aquatique les individus d'association sont très souvent disséminés en mosaïque, de ce fait, les surfaces des relevés sont souvent faibles, inférieures au $\mathrm{m}^{2}$.

- les espèces particulièrement liées à un groupe de relevés sont dites "caractéristiques" de l'association végétale définie par ce groupe de relevés; les autres espèces présentes étant qualifiées de "compagnes ». De même, nous distinguerons des espèces caractéristiques de catégories de plus en plus extensives, l'alliance, l'ordre et la classe, dont les listes spécifiques correspondent respectivement à la somme de celles des associations végétales, des alliances et des ordres.

- le pourcentage de recouvrement des espèces est noté au niveau de chaque individu d'association.

\subsection{Les composantes mésologiques}

- La hauteur d'eau ou profondeur est mesurée en $\mathrm{cm}$ au niveau du relevé, et nous faisons une moyenne dans le cas où le groupement végé- 
tal se développe à différentes profondeurs. Ce paramètre est mesuré avec la perche du micromoulinet graduée tous les $10 \mathrm{~cm}$.

- La vitesse du courant est mesurée pendant 30 secondes à $3 \mathrm{~cm}$ au-dessus de la surface d'attache de l'espèce considérée quand celle-ci nous le permet (espèce de faible dimension, flottant dans le courant parallèlement au fond ou espèce à densité pas trop élevée permettant un fonctionnement normal de l'hélice du micromoulinet), sinon nous sommes contraints de prendre les valeurs de ce paramètre aux limites de l'échantillon.

Le micromoulinet utilisé est de marque OTT, du type C 31, n 14724.

Nous avons utilisé l'hélice de $80 \mathrm{~mm}$ de diamètre, dont l'équation servant à tracer l'abaque, donnant la vitesse en $\mathrm{m} / \mathrm{s}$, est la suivante :

vitesse $(\mathrm{m} / \mathrm{s})=0,1370 \mathrm{n}+0,021$ avec $\mathrm{n}=$ nombre de t./s. pour $\mathrm{v}<1,25 \mathrm{~m} / \mathrm{s}$.

- La granulométrie du fond est relevée méthodiquement en substrat principal, secondaire et quelquefois tertiaire.

Différentes classifications dimensionnelles granulométriques des sédiments existent; nous utilisons celle de Cailleux (1954), complétée de quelques éléments :

$1:$ affleurement de la roche en place

2: tuf compact

3: $\operatorname{blocs}(>200 \mathrm{~m})$

4: cailloux, galets $(20$ à $200 \mathrm{~m})$

5 : gravette, gravillon ( 2 à $20 \mathrm{~mm}$ )

6 : sable $(0,2$ à $2 \mathrm{~mm})$

7: sablon $(0,02$ à $0,2 \mathrm{~mm})$

$8:$ limon et vase $(<0,02 \mathrm{~mm})$

9 : éléments homométriques cimentés

\section{3. - RESULTATS}

\subsection{Les associations à Phanérogames}

\subsubsection{Classe : Phragmitetea}

a) Le Phragmitetum communis (Gams, 1927) Schmale (1939).

- Physionomie : La vétégation est disposée en bandes relativement étroites de chaque côté du cours d'eau. Ce groupement végétal est caractérisé par une hauteur assez importante $(2 \mathrm{~m})$, par une densité forte.

- Composition floristique : La composition floristique globale est donnée par le tableau I. Parmi les espèces caractéristiques, Typha 
latifolia n'apparaît que dans deux relevés, Phragmites communis se comportant en espèce "sociale ", c'est-à-dire laissant peu de place pour les espèces compagnes. Seules Mentha aquatica et Helosciadium nodiflorum sont présentes dans plusieurs relevés en quantités appréciables.

- Ecologie : La hauteur d'eau dépasse rarement $30 \mathrm{~cm}$, les vitesses de courant sont nulles, le substrat se compose essentiellement de limons et de sables. Les faibles profondeurs d'eau relevées à une époque où la Loue est au-dessus de son débit d'étiage montrent que ces zones de végétation peuvent être émergées pendant de longues périodes, comme en témoigne la présence d'Urtica dioica. Nous n'avons observé cette association que dans la basse Loue.

\begin{tabular}{|c|c|c|c|}
\hline Valeurs & Minimales & Moyennes & Maximales \\
\hline $\begin{array}{l}\text { Température }\left({ }^{\circ} \mathrm{C}\right) \\
\text { Superficie }\left(\mathrm{m}^{2}\right) \\
\text { ?rofondeur }(\mathrm{cm}) \\
\text { Recouvrement en }\end{array}$ & $\begin{array}{r}6 \\
1 \\
0 \\
30\end{array}$ & $\begin{array}{r}11,9 \\
7,8 \\
15,2 \\
71,8\end{array}$ & $\begin{array}{l}14 \\
20 \\
50 \\
90\end{array}$ \\
\hline
\end{tabular}

FRÉOUENCE RECOUV. MOYEN ECART TYPE $\%$

CARACTÉRISTIQUES D'ASSOCIATION

Typha latifolia L.

CARACTÉRISTIQUES D'ORDRE ET DE ClasSE

Enanthe fistulosa $\mathrm{L}$.

Alisma plantago $\mathrm{L}$.

Iris pseudacoris L.

COMPAGNES:

Elodea canadensis Michx.

Mentha aquatica $\mathrm{L}$.

0,18

0,36

0,27

0,36

0,09

0,27
5,5

4,5

15,0

1,0

20,3

8,7

0,0

15,5

1,0

30,0

\section{b) L'Helosciadetum nodiflori Br.-Bl. 1931}

- Physionomie : C'est un groupement végétal d'espèces hélophyteshydrophytes de petite taille, dépassant l'eau de 5 à $10 \mathrm{~cm}$, et à recouvrement important.

- Composition floristique: Elle est récapitulée sur le tableau II. Nasturtium officinale et Helosciadium nodiflorum, les deux espèces caractéristiques, se comportent en espèces sociales, éclipsant la plupart des autres plantes, grâce à une multiplication végétative extrêmement abondante, par racines adventives pour le cresson et par rameaux rampants pour Helosciadium. La compagne la mieux représentée est Sparganium minimum, dans les bras morts. 
- Ecologie: Cette association atteint son meilleur développement dans les alluvions fines et dans les canaux de dérivation des moulins, pour Nasturtium officinale avec une hauteur d'eau limitée et une vitesse de courant faible mais permanente. En ce qui concerne les individus d'association dominés par Helosciadium nodiflorum, nous les trouvons sur des fonds vaso-limoneux dans une hauteur d'eau optimale de $30-40 \mathrm{~cm}$, mais pouvant atteindre $90 \mathrm{~cm}$, le courant étant toujours nul.

Nous avons regroupé dans cette association tous les relevés dominés par les deux espèces caractéristiques de l'association créée par BraunBlanquet en 1931, mais il convient de noter que ces deux espèces s'excluent mutuellement et que l'on pourrait fort bien délimiter, comme le fait J. V. Wattez (1975), citant E. Oberdorfer (1970), deux associations totalement différentes, l'une, le Nasturtietum officianalis, Seibert (1962) étant beaucoup plus rhéophile que l'Helosciadium nodi. flori.

Tableau II. - Phragmitetea Tx. et Prsg. 1942

Phragmitetalia (Koch 26) Tx. et Prsg. 1942

GLYCERIO - SPARGANION Br. - Bl. et Siss 1942

HELOSCIADIUM NODIFLORI Br. - Bl. 1931.

Nombre de relevés : 11; Vitesse $(\mathrm{m} / \mathrm{s}): 0$; Substrat : 8 - 4-7.

\begin{tabular}{|c|c|c|c|}
\hline Valeurs & Minimales & Moyennes & Maximales \\
\hline Température $\left({ }^{\circ} \mathrm{C}\right)$ & 6 & 11,9 & 14 \\
\hline Superficie $\left(\mathrm{m}^{2}\right)$ & 1 & 2,9 & 6 \\
\hline Profondeur $(\mathrm{cm})$ & 10 & 35,5 & 90 \\
\hline \multirow[t]{2}{*}{ Recouvrement en \% } & 100 & 75,5 & 40 \\
\hline & FRÉQUENCE & $\begin{array}{l}\text { RECOUV. MOYEN } \\
\%\end{array}$ & ECART TYPE \\
\hline \multicolumn{4}{|l|}{ CARACTÉRISTIQUES D'ASSOCIATION } \\
\hline $\begin{array}{l}\text { Helosciadium nodiflorum Koch } \\
\text { Nasturtium officinale } \mathrm{R} . \mathrm{Br} \text {. }\end{array}$ & $\begin{array}{l}0,73 \\
0,27\end{array}$ & $\begin{array}{l}46,3 \\
60,0\end{array}$ & $\begin{array}{l}24,0 \\
21,6\end{array}$ \\
\hline \multicolumn{4}{|l|}{ CARACTÉRISTIQUES D'ALLIANCE } \\
\hline $\begin{array}{l}\text { Veronica beccabunga L. } \\
\text { CARACTÉRISTIOUES D'ORDRE ET DE CLASSE }\end{array}$ & 0,09 & 10,0 & 0,0 \\
\hline Enanthe fistulosa & 0,18 & 10,0 & 0,0 \\
\hline Alisma plantago $\mathrm{L}$. & 0,09 & 1,0 & 0,0 \\
\hline \multicolumn{4}{|l|}{ COMPAGNES } \\
\hline Sparganium minimum Fries & 0,36 & 7,8 & 3,9 \\
\hline Elodea canadensis Michx. & 0,36 & 5,5 & 4,5 \\
\hline Callitriche sp. & 0,18 & 25,0 & 15,0 \\
\hline Mentha aquatica $\mathrm{L}$. & 0,18 & 5,5 & 4,5 \\
\hline Baldingera arundinacea Dumort. & 0,18 & 10,0 & 0,0 \\
\hline Fotamogeton fluitans Roth. & 0,18 & 15,0 & 5,0 \\
\hline Potamogeton densus $\mathrm{L}$. & 0,18 & 25,0 & 15,0 \\
\hline Ranuncullus trichophyllus chaix & 0,09 & 10,0 & 0,0 \\
\hline Polygonum amphibium $\mathbf{L}$. & 0,09 & 10,0 & 0,0 \\
\hline Ranunculus circinatus Sibth & 0.09 & 1,0 & 0,0 \\
\hline
\end{tabular}




\subsubsection{Classe : Potamogetonetea}

\section{a) Le Callitricho-Myriophylletum alterniflori}

- Physionomie: Si la superficie des individus d'association n'est pas très grande, le \% de recouvrement est important, certains Callitriche formant de véritables tapis à la surface de l'eau grâce à leurs rosettes de feuilles terminales. La floraison est très discrète, les fleurs minuscules se développant sous l'eau.

- Composition floristique : Le tableau III donne la composition floristique de cette association.

Nous remarquons la pauvreté spécifique de ce groupement, due en particulier à une reproduction rapide des Callitriches freinant le développement des autres espèces.

- Ecologie: Cette association apparaît dès que la Loue perd son caractère torrentiel, en eau calme peu profonde $(30$ à $50 \mathrm{~cm})$, sur fonds constitués de limons et de vase; des débris végétaux étant fréquents.

Ces secteurs correspondent aux bordures de barrages et aux arrivées des canaux de dérivation des moulins, ce groupement se trouvant alors plus ou moins en mélange avec l'association à Nasturtium officinale.

Tableau III. - Potamogetonetea Tx. et Prsg. 1942

Potamogetonetalia Koch 1942

RANUNCULION FLUITANTIS Neuhsg 1959

CAILITRICHO - MYRIOPHYLLETUM ALTERNIFLORI Steusloff 1939.

Nombre de relevés : 7 ; Vitesse $(\mathrm{m} / \mathrm{s}): 0$; Substrat : 8 . 7 .

\begin{tabular}{|c|c|c|c|}
\hline Valeurs & Minimales & Moyennes & Maximales \\
\hline Température $\left({ }^{\circ} \mathrm{C}\right)$ & 11 & 12,4 & 14 \\
\hline Superficie $\left(\mathrm{m}^{2}\right)$ & 1 & 5,3 & 20 \\
\hline Profondeur $(\mathrm{cm})$ & 30 & 45,7 & 80 \\
\hline Recouvrement en $\%$ & 50 & 75,0 & 85 \\
\hline
\end{tabular}

FRÉQUENCE RECOUV. MOYEN ECART TYPE $\%$

Caractéristiques d'association

Callitriche hamulata Lam.

CARACTÉRISTIQUES D'ORDRE ET DE CLASSE

Elodea canadensis Michx.

Compagnes

Ranunculus trichophyllus chaix

Ranunculus circinatus Sibth.

Veronica anagallis $\mathrm{L}$.

Myriophyllum verticillatum $\mathbf{L}$.

Sparganium minimum Fries

Baldıngera arundinacea Dumort.

$\begin{array}{lrr}\mathbf{1 , 0} & \mathbf{5 8 , 6} & \mathbf{1 7 , 3} \\ & & \mathbf{3 , 8} \\ 0,57 & 6,5 & \\ & & \mathbf{5 , 0} \\ 0,29 & \mathbf{1 5 , 0} & \mathbf{0 , 0} \\ 0,29 & 10,0 & \mathbf{0 , 0} \\ 0,14 & 10,0 & \mathbf{0 , 0} \\ 0,14 & 1,0 & \mathbf{0 , 0} \\ 0,14 & 1,0 & \mathbf{0 , 0} \\ 0,14 & 10,0 & \end{array}$


b) Le Potamogetonetum perfoliati-lucentis Wilzek 1935.

- Physionomie : Le nombre de relevés correspondant à cette association est peu élevé. Le recouvrement est relativement faible, dépassant rarement $50 \%$. Potamogeton lucens apparaît comme l'espèce dominante. Le groupement est composé presque uniquement d'espèces hydrophytes, complètement immergées ou à feuilles flottant à la surface de l'eau.

- Composition floristique : Elle est donnée par le tableau IV. Cette association est caractérisée par $P$. lucens et $P$. perfoliatus. Le nombre d'espèces compagnes est limité, les bryophytes ont disparu et seul le genre Vaucheria subsiste chez les algues.

- Ecologie : Les endroits où l'on rencontre cette association sont caractérisés par une profondeur assez importante $(60$ à $100 \mathrm{~cm})$, une vitesse d'écoulement nulle et un substrat à base de vase, limon et sablon. Ce sont des zones de dépôts dans les retenues de barrages ou dans les bras morts, dans la moyenne et la basse Loue.

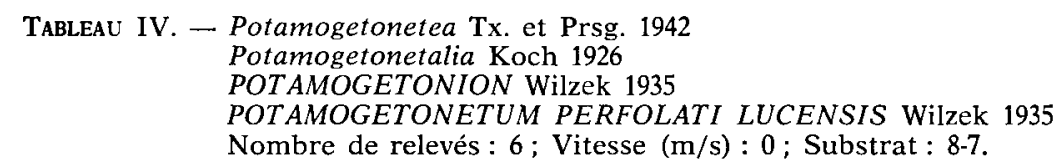

\begin{tabular}{lccc}
\hline Valeurs & Minimales & Moyennes & Maximales \\
\hline Température $\left({ }^{\circ} \mathrm{C}\right)$ & $\mathbf{1 2}$ & 13,3 & 14 \\
Superficie $\left(\mathrm{m}^{2}\right)$ & 1 & 9,8 & 30 \\
Profondeur $(\mathrm{cm})$ & $\mathbf{2 0}$ & 70,0 & 100 \\
Recouvrement en \% & $\mathbf{1 5}$ & 31,7 & 60 \\
\hline
\end{tabular}

FrÉQuence Recouv. MOYEN ECART TYPE $\%$

CARACTÉRistiques d'AsSociation

Potamogeton lucens $\mathrm{L}$.

Potamogeton perfoliatus $\mathrm{L}$.

Callitriche hamulata Lam.

CARActéristiques d'Alliance

Ranunculus fluitans Lam.

COMPAGNES

Roripa amphibia (L.) Besser

Baldingera arundinacea Dumort.

Polygonum amphibium L.

Vaucheria sp.

0.17

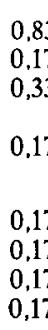

27,0

10,0

5,5

1,0

20,0

10,0

10,0

5,0
12,5

0,0

4,5

0,0

0,0

0,0

0,0

0,0

c) Le Ranunculetum fluitantis All. 1922

- Physionomie: Cette association végétale est composée uniquement de plantes hydrophytes solidement fixées au substrat, flottant dans le courant, certaines atteignant la surface de l'eau afin de fleurir au-dessus. 
Le recouvrement est généralement fort, la superficie des individus d'association pouvant être très importante si les conditions sont propices; une bonne partie de la Loue devenant blanche des fleurs de Ranunculus fluitans au mois de juin.

- Composition floristique : La diversité floristique est limitée, les individus d'association sont souvent monospécifiques avec Ranunculus fluitans faisant figure d'espèce sociale. Parmi les compagnes, nous pouvons trouver quelquefois des mousses ou des algues se développant sur les quelques galets disséminés dans le substrat dominant. (Tableau V).

- Ecologie : La granulométrie la plus fréquente est assez fine, à dominante sablo-limoneuse. La condition d'implantation de l'association semble être la présence des sables, car nous ne la retrouvons jamais sur un substrat uniquement composé de vase et de limons, ce qui correspond bien avec les fourchettes de vitesses du courant qui entraîne la plupart des éléments très fins. Ce groupement est donc lié à la présence permanente d'un écoulement de l'eau, dont la vitesse peut être forte en certains endroits, notamment sur les barrages où l'association peut s'implanter quelquefois.

Le Ranunculetum fluitantis peut donc être qualifié de rhéophile, préférant les vitesses de l'ordre de $0,3 \mathrm{~m} / \mathrm{s}$ en débit moyen ce qui cor-

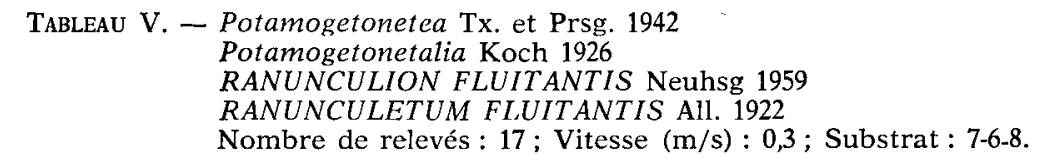

\begin{tabular}{|c|c|c|c|}
\hline Valeurs & Minimales & Moyennes & Maximales \\
\hline Température $\left({ }^{\circ} \mathrm{C}\right)$ & 5,5 & 10,9 & 13 \\
\hline Superficie $\left(\mathrm{m}^{2}\right)$ & 0,5 & 23,5 & 150 \\
\hline Profondeur $(\mathrm{cm})$ & 30,0 & 61,8 & 100 \\
\hline \multirow[t]{2}{*}{ Recouvrement en \% } & 5,0 & 53,2 & 100 \\
\hline & FRÉqueNCE & $\begin{array}{l}\text { RECOUV. MOYEN } \\
\%\end{array}$ & ECART TYPE \\
\hline \multicolumn{4}{|c|}{ CARACTÉRISTIQUES D'ASSOCIATION ET D'ALLIANCE } \\
\hline Ranunculus fluitans Lmk. & 1,00 & 42,1 & 29,2 \\
\hline \multicolumn{4}{|l|}{ CaRactéristiques d'oRdRe eT DE Classe } \\
\hline Elodea canadensis Michx. & 0,06 & 20,0 & 0,0 \\
\hline Myriophyllum spicatum L. & 0,66 & 30,0 & 0,0 \\
\hline Potamogeton densus $\mathrm{L}$. & 0,06 & 60,0 & 0,0 \\
\hline \multicolumn{4}{|l|}{ COMPAGNES } \\
\hline Fontinalis antipyretica $\mathrm{L}$. & 0,18 & 8,7 & 5,9 \\
\hline Cinclidotus aquaticus (Jaeg.) B.e. & 0,06 & 10,0 & 0,0 \\
\hline Vaucheria sp. & 0,06 & 5,0 & 0,0 \\
\hline Cladophora sp. & 0,06 & 10,0 & 0,0 \\
\hline Phormidium sp. & 0,06 & 30,0 & 0,0 \\
\hline
\end{tabular}


respond bien aux profondeurs les plus fréquentes $(60$ à $70 \mathrm{~cm})$; la fourchette de hauteurs d'eau allant de 30 à $100 \mathrm{~cm}$.

Les individus d'association se rencontrent à partir de Mouthier jusqu'à la confluence avec le Doubs ; leur absence dans le cours torrentiel pouvant être expliquée par la trop grande vitesse de l'eau entraînant un défaut de substrat adéquat, ainsi que par un manque de luminosité.

d) Le Myriophyllo - Nupharetum Koch 1926

- Physionomie: Les taches constituées par Nuphar luteum à la surface des eaux de la Loue ne sont jamais très étendues. Elles sont localisées dans les endroits abrités du courant, vers le bord, ou au milieu des bras morts lorsque la profondeur de l'eau est suffisante.

- Composition floristique : Elle est récapitulée sur le tableau VI.

Nous pouvons remarquer que ce groupement tend vers la monospécificité ; seule, parmi les caractéristiques d'association, Nuphar luteum étant représentée, Nymphea alba et Myriophyllum verticillatum étant totalement absentes. Parmi les compagnes, nous trouvons surtout des hydrophytes complètement immergés, seules les feuilles de Sparganium minimum pouvant flotter en surface en compagnie de celles du nénuphar jaune.

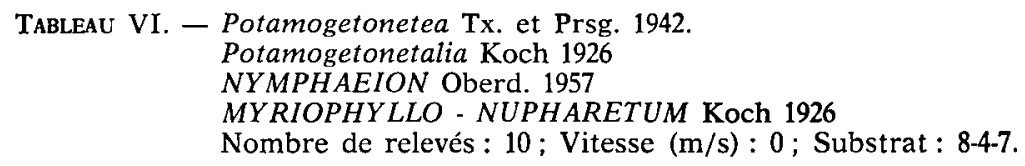

\begin{tabular}{|c|c|c|c|}
\hline Valeurs & Minimales & Moyennes & Maximales \\
\hline Température $\left({ }^{\circ} \mathrm{C}\right)$ & 12 & 12,6 & 13 \\
\hline Superficie $\left(\mathrm{m}^{2}\right)$ & 2 & 22,7 & 100 \\
\hline Profondeur $(\mathrm{cm})$ & 50 & 93,0 & 150 \\
\hline Recouvrement en \% & 20 & 49,5 & 90 \\
\hline
\end{tabular}

FrÉOUENCE RECOUV, MOYEN ECART TYPE $\%$

CARACTÉRISTIQUES D'ASSOCIATION ET D'AlLIANCE

Nuphar luteum Sm.

1,00

0,10

CARACTÉRISTIQUES D'ORDRE ET DE CLASSE

Myriophyllum spicatum L.

Potamogeton densus L.

COMPAGNES

Sparganium minimum Fries

Hottonia palustris L.

Helosciadium nodiflorum Koch

Enanthe fistulosa L.

Ranunculus trichophyllus chaix

Potamogeton fluitans Roth

Typha latifolia L.
0,10

0,30

0,20

0,20

0,10

0.10

0,10

0,10
31,0

10,0

10,0

11,7

20,0

25,0

10,0

10,0

10,0

10,0
15,6

0,0

0,0

6,2

0,0

15,0

0,0

0,0

0,0

0,0 
- Ecologie : Cette association apparaît en eau profonde (70 à $150 \mathrm{~cm}$ ) et stagnante, sur un substrat vaso-limoneux, recouvrant quelquefois des galets.

Nous n'avons trouvé des individus la représentant que dans la partie basse de la Loue.

\subsection{Les associations à Bryophytes}

\subsubsection{Classe : Montio-Cardaminetea}

a) Le Cratoneuretum communati Walther 1942

- Physionomie : C'est une association d'espèces rampantes sur un substrat compact, avec certaines espèces incrustantes. Les individus d'association rencontrés ont, en général, une faible surface.

- Composition floristique: Elle est récapitulée sur le tableau VII.

Seules des Bryophytes et quelques algues sont présentes. Les hépatiques à thalle et les Cratoneurum dominent; les caractéristiques d'ordre et de classe étant peu représentées.

TableaU VII. - Montio - cardaminetea Br. Bl. et Tx. 1943

Montio - cardaminetalia Rawl. 1928

CRATONEURION COMMUTATI Koch 1936

CRATONEURETUM COMMUTATI Walter 1942

Nombre de relevés : 8 ; Vitesse $(\mathrm{m} / \mathrm{s}): 0$; Substrat : 3-9.

\begin{tabular}{lccc}
\hline Valeurs & Minimales & Moyennes & Maximales \\
\hline Température $\left({ }^{\circ} \mathrm{C}\right)$ & 12 & 12,0 & 12 \\
Superficie $\left(\mathrm{m}^{2}\right)$ & 0,4 & 3,2 & 15 \\
Profondeur $(\mathrm{cm})$ & 0 & 0,3 & 1 \\
Recouvrement en \% & 90 & 67,5 & 50 \\
\hline
\end{tabular}

FRÉQUENCE RECOUV. MOYEN ECART TYPE $\%$

CaRactéristiques D'AsSociation

Pellia fabbroniana Raddi

Conocephalum conicum (L.) Dum.

Cratoneurum filicinum (Hedw.) Roth.

CARACTÉRISTIQUES D'Alliance

Cratoneurum commutatum (Hedw.) Roth.

Compagnes

Fontinalis antipyretica $\mathrm{L}$.

Platyhypnidium rusciforme (Neck.) Fleisch

Leptodyctium riparium Wainst.

Cinclidotus fontinalö̈des (Hedw.) P. Beauv.

Marchantia polymorpha $\mathrm{L}$.

Fissidens crassipes Wils.

Rhynchostegiella curviseta (Brid.)

Cinclidotus aquaticus (Jaeg.) Br. Limpr.

Phormidium sp.

Nostoc sp.

Porella platyphylla (L.) Lindb.
0,38

0,50

0,50

20,0

32,5

22,8

14,1

12,9

22,5

$0,25 \quad 35,0 \quad 5,0$

0,38

0,13

0,13

0,50

0,50

0,25

0,13

0,13

0,13

0.13

0,13
5,0

$10,3 \quad 7,8$

$1,0 \quad 0,0$

$1,0 \quad 0,0$

$7,8 \quad 3,9$

$17,5 \quad 8,3$

$10,0 \quad 0,0$

$10,0 \quad 0,0$

$10,0 \quad 0,0$

$10,0 \quad 0,0$

$1,0 \quad 0,0$

$10,0 \quad 0,0$ 
- Ecologie: Ce groupement végétal prospère sur les blocs et les parois verticales aspergés ou suintants. Ce n'est pas véritablement une association aquatique car on ne la rencontre jamais sur des substrats propices toujours immergés.

Ces conditions spéciales d'habitat font qu'on peut la rencontrer tout le long de la Loue.

b) Le Cinclidotetum fontinaloïdis Gams 1927

- Physionomie : L'association à Cinclidotus fontinalö̈des est caractérisée par un recouvrement important, formant des tapis vert foncé de quelques centimètres d'épaisseur sur le substrat, les tiges de mousses atteignant au maximum $15 \mathrm{~cm}$ et flottant dans le courant.

- Composition floristique : Elle est récapitulée sur le tableau VIII.

Les individus d'association sont assez souvent monospécifiques, les caractéristiques d'ordre et de classe sont peu représentées, seule $\mathrm{Bra}$ chythecium rivulare formant quelques taches claires dans le groupement vert foncé. Parmi les compagnes, c'est le genre Vaucheria qui est le plus souvent présent, Hydrurus foetidus apparaissant sur les barrages.

TABLEAU VIII. - Fontinaletea antipyreticae V. Hübschmann 1957

Fontinaletalia antipyreticae V. Hübschmann 1957

CINCLIDOTO-FISSIDENTION V. Hübschmann 1957

CINCLIDOTETUM FONTINALOIDIS Gams. 1927

Nombre de relevés : 13 ; Vitesse $(\mathrm{m} / \mathrm{s}): 0,3$; Substrat : 3-1-9.

\begin{tabular}{lccc}
\hline Valeurs & Minimales & Moyennes & Maximales \\
\hline Température $\left({ }^{\circ} \mathrm{C}\right)$ & 5,5 & 9,4 & 12 \\
Superficie $\left(\mathrm{m}^{2}\right)$ & $\mathbf{0 , 2}$ & $\mathbf{1 1 , 0}$ & 50 \\
Profondeur $(\mathrm{cm})$ & $\mathbf{0 , 0}$ & $\mathbf{1 2 , 7}$ & 40 \\
Recouvrement en \% & 50,0 & $\mathbf{7 2 , 3}$ & 90 \\
\hline
\end{tabular}

Fréquence Recouv. MOYEN Ecart type $\%$

CARACTÉRISTIQUES D'ASSOCIATION

Cinclidotus fontinaloïdes (Hedw.) P. Beauv. 1,00

Caractéristiques d'alliance

Fissidens crassipes Wils.

$0,08 \quad 10,0 \quad 0,0$

CARACTÉRISTIQUES D'ORDRE ET DE CLASSE

Fontinalis antipyretica $\mathrm{L}$.

Leptodyctium riparium Wainst.

$0,08 \quad 10,0$

$0,15 \quad 6,0$

0,0

COMPAGNES

Ranunculus fluitans Lam.

Hydrurus foetidus (Vill.) Trév.

Cinclidotus aquaticus (Jaeg.) B.e.

Brachythecium rivulare B.e.

Phormidium autumnale (Ag.) Gomont

Cladophora sp.

0,15

0,08

0,08

0,15

0,08

Vaucheria sp.

0,15

0,46 
- Ecologie : Le substrat se compose de blocs et de galets, de barrages, d'affleurements de la roche ou même de tufs. La vitesse du courant peut être nulle ou très forte, l'optimum se situant entre 0,2 et $0,6 \mathrm{~m} / \mathrm{s}$. La fourchette de hauteur d'eau est grande $(0$ à $90 \mathrm{~cm})$. Les individus d'association situés dans des faibles hauteurs d'eau seront exondés pendant les débits d'étiage et résistent donc bien à une émersion prolongée. A. Empain (1974) considère Cinclidotus fontinaloüdes comme " recherchant une émersion prolongée mais sans excès ».

L'association apparaît en quantité importante à partir de Mouthier, dès que la Loue perd son caractère torrentiel et que la Iuminosité devient plus importante ; elle prospère jusqu'à la confluence avec le Doubs, sur les blocs d'enrochement, depuis le rajeunissement artificiel créé par le redressement de la partie inférieure de la Loue.

c) Le Cincliaotetum aquatici A. V. Hübschmann et Philippi 1956.

- Physionomie : C'est l'association type de la vallée supérieure de la Loue, abondamment présente sur les blocs et les affleurements rocheux. Elle se présente comme un tapis vert foncé presque ininterrompu sur les surfaces qu'elle peut coloniser.

- Composition floristique : Elle est récapitulée sur le tableau IX.

L'espèce dominante et caractéristique de l'association est Cinclidotus aquaticus, que l'on peut qualifier de sociale. En effet, elle est fréquemment seule; la compagne la mieux représentée étant la minuscule Rhynchostegiella curviseta. Les caractactéristiques d'alliance, d'ardre, et de classe, sont présentes sporadiquement. La seule phanérogame pouvant s'implanter dans les sédiments déposés entre les blocs sur les bannages est Ranunculus fluitans.

- Ecologie: La profondeur peut atteindre $60 \mathrm{~cm}$, mais l'optimum est de l'ordre de $20 \mathrm{~cm}$, ce qui correspond en général à des vitesses d'écoulement assez élevées $(0,5$ à $1 \mathrm{~m} / \mathrm{s})$. Il semble en fait que l'association puisse prospérer dans des zones assez calmes et fréquemment émergées, les relevés ayant été faits en période d'eaux assez hautes, et les zones les moins profondes devant être exondées en débits moyens et d'étiage.

Le substrat type est composé de blocs ou d'affleurements rocheux. L'ensoleillement est assez faible tout le long de son habitat, la Loue circulant alors sous un tunnel d'arbres. L'association est vite limitée après Mouthier. Elle est alors remplacée par le Cinclidotetum fontinaloïdes, les deux espèces caractéristiques de chaque association s'excluant mutuellement presque toujours.

Nous n'avons fait qu'un seul relevé dans la basse Loue où Cinclidotus aquaticus était significativement présent sur les barrages de tufs à Lombard. 
TABLEAU IX. - Fontinaletea antipyreticae V. Hübschmann 1957 Fontinaletalia antipyreticae $\mathrm{V}$. Hübschmann 1957 CINCLIDOTO - FISSIDENTION V. Hübschmann 1957 CINCLIDOTETUM AQUATICI V. Hübschmann et Philippi 1956 Nombre de relevés : 16 ; Vitesse $(\mathrm{m} / \mathrm{s}): 0,7$; Substrat : 3-4-1.

\begin{tabular}{|c|c|c|c|}
\hline Valeurs & Minimales & Moyennes & Maximales \\
\hline Température $\left({ }^{\circ} \mathrm{C}\right)$ & 5,5 & 7,2 & 10 \\
\hline Superficie $\left(\mathrm{m}^{2}\right)$ & 0,5 & 30,3 & 150 \\
\hline Profondeur $(\mathrm{cm})$ & 0,0 & 21,9 & 60 \\
\hline \multirow[t]{2}{*}{ Recouvrement en \% } & 30,0 & 72,5 & 100 \\
\hline & FRÉQUENCE & $\underset{\%}{\text { RECOUV. MOYEN }}$ & ECART TYPE \\
\hline CARACTÉRISTIOUES D'ASSOCIATION & & & \\
\hline $\begin{array}{l}\text { Cinclidotus aquaticus (Jaeg.) B.e. } \\
\text { CARACTÉRISTIQUES D'ALLIANCE }\end{array}$ & 1,00 & 58,1 & 17,1 \\
\hline Fissidens crassipes Wils. & 0,06 & 10,0 & 0,0 \\
\hline CARACTÉRISTIQUES D'ORDRE ET DE CLASSE & & & \\
\hline $\begin{array}{l}\text { Leptodyctium riparium Warnst. } \\
\text { COMPAGNES }\end{array}$ & 0,31 & 10,2 & 8,1 \\
\hline Ranunculus fluitans Lam. & 0,13 & 25,0 & 15,0 \\
\hline Cratoneurum commutatum (Hedw.) Roth. & 0,06 & 1,0 & 0,0 \\
\hline Rhynchostegiella curviseta Brid. Limpr. & 0,13 & 40,0 & 10,0 \\
\hline $\begin{array}{l}\text { Brachythecium rivulare B.e. } \\
\text { Chiloscyphus polyanthus var. rivularis }\end{array}$ & 0.06 & 5,0 & 0,0 \\
\hline (Schrad.) Nees. & 0,13 & 7,5 & 7,5 \\
\hline Phormidium autumnale (Ag.) Gomont. & 0,06 & 1,0 & 0,0 \\
\hline Cladophora sp. & 0,06 & 1,0 & 0,0 \\
\hline
\end{tabular}

\section{CONCLUSIONS}

De cette étude sommaire nous pouvons tirer les caractères principaux de la végétation de l'écosystème Loue. Après une reconnaissance des groupements végétaux existants, il est possible, à l'aide de mesures effectuées, de définir les variations des composantes morphodynamiques de chaque association. Ces composantes sont étroitement liées et dépendantes les unes des autres, c'est-à-dire qu'elles varient simultanément.

Le tableau $\mathrm{X}$ résume "l'écologie " et le préférendum typologique de chacun des groupements. Certaines associations possèdent un préférendum typologique longitudinal nettement défini, c'est le cas du Myriophyllo-Nupharetum et du Potamogetonetum aquatici pour la Haute Loue. Au contraire, la plupart des autres groupements n'ont aucun préférendum typologique longitudinal, ils se rencontrent sur tout le parcours de la rivière dès que sont réunies les conditions écologiques nécessaires (typologie stationnelle). 


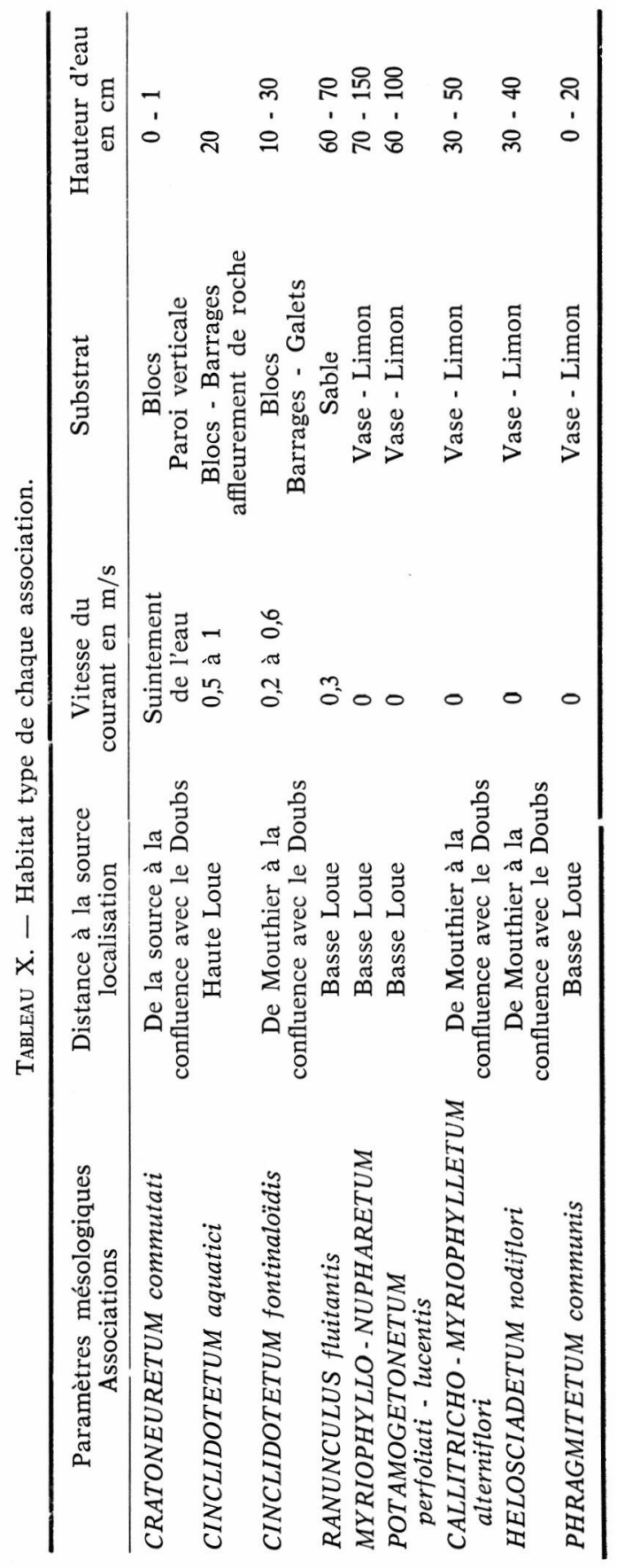




\section{TRAVAUX CITES}

Allorge (A. P.). 1921-1922. - Les associations végétales du Vexin Français. Revue générale de Bota., XXXIII.XXXIV, $\mathrm{n}^{\circ} 393-408$.

Baillaud (R.). 1955-1957. - Climatologie comtoise et jurasienne. Ann. Sci. Univ. Besançon, 1:1-31, 2:1-41, 3:1-69.

Delarozière (O.). 1968. - Contribution à l'étude du Bassin du Doubs. Climatologie, hydrologie et déficit d'écoulement rapportés aux unités géologiques. Thèse de $3^{e}$ cycle, Fac. Sci. Univ. Besançon. G. R. Jura-Alpes, Lyon, 100 p.

Descy (J.P.). 1973. - La végétation algale benthique de la Meuse belge et ses relations avec la pollution des eaux. Lejeunia, $66: 1-62$.

Descy (J. P.). 1976. - La végétation algale benthique de la Somme et ses relations avec la pollution des eaux. Mém. Soc. Roy. Bot. Belg., 7 : 101-128.

Dreyfuss (M.), Théobald (N.) et Thiebaut (J.). 1962. - Vue d'ensemble sur le Jura Franc-Comtois et la retombée méridionale des Vosges. Ann. Sci. Univ. Besançon, Géol. 16 : 93-112.

Empain (A.). 1973. - La végétation bryophytique aquatique et subaquatique de la Sambre belge, son déterminisme écologique et ses relations avec la pollution des eaux. Lejeunia, $69: 1-58$.

Empain (A.). 1974. - Relations quantitatives entre les Bryophytes de la Sambre belge et leur fréquence d'émersion. Distribution verticale et influence de la pollution. Bull. Soc. Roy. Bot. Belgique, 107: 361-374.

Fournier (E.). 1900. - Les réseaux hydrographique du Doubs et de la Loue dans leurs rapports avec la structure géologique. Ann. Géogr., 9 (45) : 219-227.

Guinochet (M.). 1973. - Phytosociologie, Masson (Collection d'Ecologie), 227 p.

Oberdorfer (E.). 1970. - Phytosoziologische Exkursionflora für Süddeutschland, p. 31 et 664 .

SELL (Y.). 1965. - Les plantes aquatiques au sens large. Mise au point d'une classification écomorphologique. Bull. Ass. Phil. d'Alsace et de Lorraine, tome XII, i : $58-65$.

Tronchet (A.). 1955. - Paysages botaniques et groupements végétaux du Jura Central. Ann. Sci. Univ. Besançon, Bota. 6 (1) : 19-44.

Verneaux (J.). 1973. - Recherches écologiques sur le réseau hydrographique du Doubs. Essai de biotypologie. Ann. Sci. Univ. Besançon, 9: $260 \mathrm{p}$.

Verneaux (J.) et Rezzouk (M.). 1974. - Les structures d'un grand cours d'eau à Salmonidés: la Loue. Annls Limnol., 10 (2) : 131-162.

WarteZ (J.R.). 1975. - Etude phytosociologique des peuplements d'Apium nodiflorum (L.) et de Nasturtium officinale (R. B.) dans le nord de la France. Documents phytosociologiques. Facs. 9-14.

Weimer-Chofardet (M.). 1946. - Etude du climat bisontin déduite de 60 années d'observations (1/12/1884-30/11/1944). Ann. Sci. Franche-Comté, 1 : 71-101. 